\title{
Transmission of light by a single subwavelength cylindrical hole in metallic films
}

\author{
Ming Bai and N. García \\ Laboratorio de Física de Sistemas Pequeños y Nanotecnología, Consejo Superior de Investigaciones \\ Científicas, Serrano 144, Madrid 28006, Spain
}

(Received 12 July 2006; accepted 12 August 2006; published online 3 October 2006)

\begin{abstract}
This letter presents theory and finite-difference time-domain calculations in excellent agreement with experiments for the transmission of a single subwavelength cylindrical hole in Ag metallic films. The authors discuss the very large enhancement in transmission by single holes in terms of surface plasmons at the interface of the hole. These are similar to the surface plasmons in metallic cylinders reported by Economou and co-workers [Phys. Rev. B 10, 3038 (1974); 24, 6908 (1981)]. The peak structures observed in experiments with arrays of holes are a consequence of interference of the beams transmitted by each hole and are determined by the hole periodicities. The role of the arrays in enhancement of the transmission due to surface plasmon polaritons appears to be marginal. (C) 2006 American Institute of Physics. [DOI: 10.1063/1.2358210]
\end{abstract}

Transmission of radiation by subwavelength holes is an interesting phenomenon that affects electron, acoustic, and electromagnetic waves. Interesting experiments ${ }^{1}$ were reported showing that the transmission of light through subwavelength holes drilled periodically in a metallic film of $\mathrm{Ag}$ was extraordinary, tens of thousands times larger, as compared with the transmission by one single hole of the same size in the same material. Recent experiments ${ }^{2}$ by part of the same team (Lezec and Thio) appear to contradict the results reported in Ref. 1 . The explanation of the experiments ${ }^{1}$ was based on the surface plasmon polaritons (SPPs) that are excited in the case of a set of periodic holes. ${ }^{3}$ The recent paper by Lezec and Thio, ${ }^{2}$ reviewing the field and containing many new data, presents a different point of view for understanding the transmission. The paper ${ }^{2}$ does not support the interpretation of SPP to understand the experimental results and disclaims the entire picture of the large transmission being due to periodic arrays of holes. Even more, they showed experimentally that the transmission enhancement by a periodic array with respect to that of a single hole is at most a factor of 7, not a factor of tens of thousands, and that there can be depression of the relative transmission as well. For more references the reader is addressed to Ref. 2 .

Although the experiments in Refs. 1 and 2 are consistent among themselves, there seems to be a disagreement in the way the data are discussed. Maybe there is a point of broken physical argument in the analysis of the data in Ref. 1 The authors compared the transmission by each hole of the array with the transmission of a single hole reported in an earlier paper by Bethe, ${ }^{4}$ and this gives enhancements of tens of thousands. However, this paper ${ }^{4}$ was a theoretical study showing that the transmission of a subwavelength hole drilled in a screen (thickness of the metal film tending to zero) of perfect metal, ideal conductor, behaves as $(r / \lambda)^{4}$, where $\lambda$ is the wavelength of the radiation and $r$ is the hole radius. But Ref. 4 is not adequate for describing the behavior of a real metal such as Ag. Also, Bethe in his work used the approximation that the field is constant through the hole but is not appropriate for holes with radius larger than $50 \mathrm{~nm}$ and

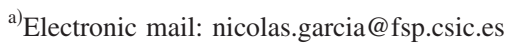

wavelengths smaller than $800 \mathrm{~nm}$. Therefore it is necessary and interesting to discuss and understand these experiments first of all for single holes. Once we understand problem for a single hole the periodic arrays can be also treated.

The aim of this letter is to study the transmissions of a hole of subwavelength size in a flat metallic film with thickness of the order of the hole diameter, as those used in the experiments. ${ }^{2}$ The transmissions can be of the order of tens of thousands times larger than the same hole in an ideal metal when simplistic estimations are considered. The understanding of this phenomenon may be done in terms of surface plasmons excited at the walls which define the hole and have the same nature as those described by Pfeiffer et al. ${ }^{5}$ and also by Martinos and Economou ${ }^{6}$ for metallic cylinders. Those are surface plasmons located at the surface on the cylinders and propagating along its axis.

In order to proceed with our work we perform frequency dependent finite-difference time-domain (FDTD) calculations for a circular hole structure on a flat metallic surface with a given diameter $d$ and thickness $t$. The metals modeled in the calculation have frequency dependent permittivities from experimental data by Johnson and Christy ${ }^{7}$ and from Physics Data. ${ }^{8}$ In the simulation, a plane pulse wave with a broad band is set as the incident wave. We recorded the transmitted wave through the structure. The frequency response of the structure is calculated by dividing the spectrum of the transmitted wave by the spectrum of the incident wave. The perfect matched layer absorbing boundary condition $^{9}$ is applied in FDTD calculation for a single hole transmission, and periodical boundary condition is applied for hole array transmission. The grid and time step are taken small enough to obtain convergent solutions.

First we performed the numerical simulations for single holes with diameters $d=200,250$, and $270 \mathrm{~nm}$ in Ag film of thickness $t=340 \mathrm{~nm}$ in order to compare with the existing experimental data [Ref. 2, Fig. 2(c)]. The incidence is normal to the surface of the metal film. In Figs. 1(a) and 1(b) we plot the transmission of single holes with different diameters. The transmission is defined as the intensity transmitted by the hole divided by the intensity arriving at the hole area $\left(\pi d^{2} / 4\right)$ versus the incident light wavelength $\lambda$. As can be 

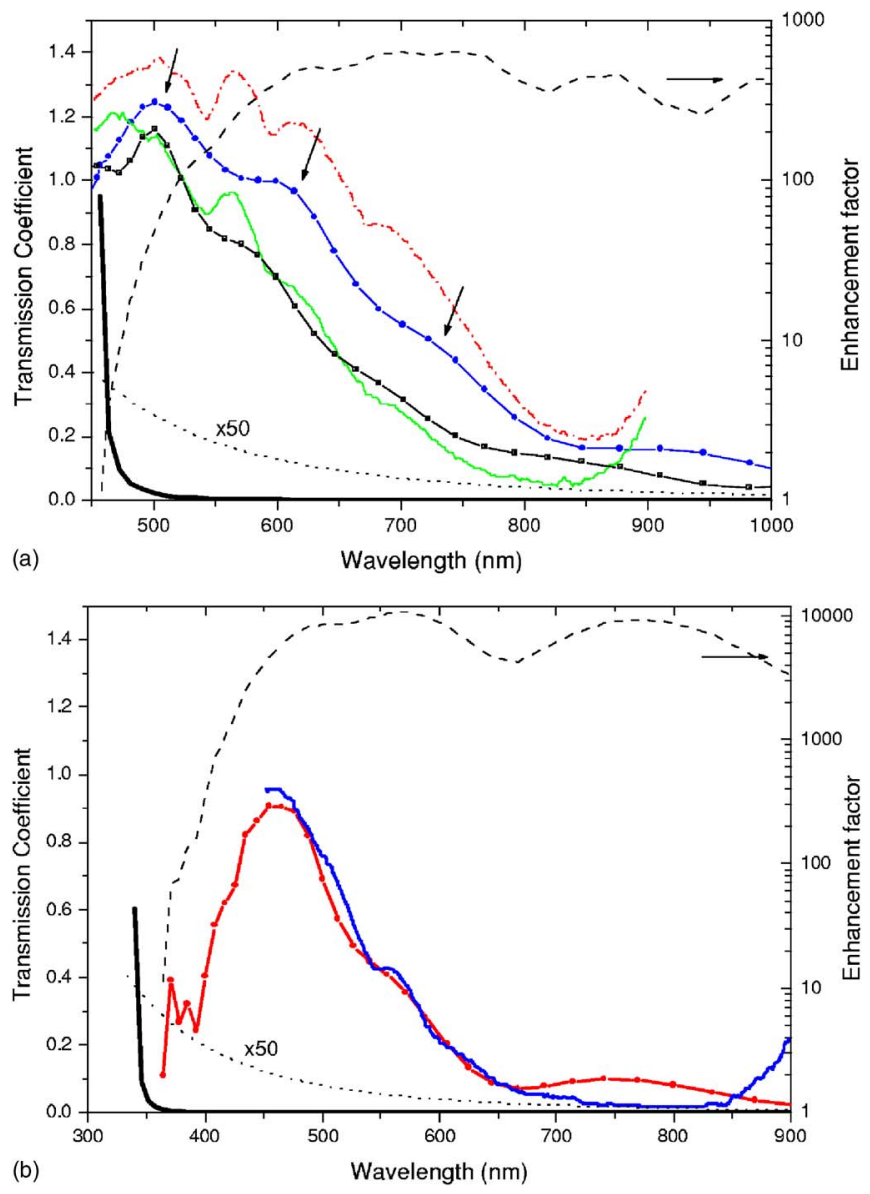

FIG. 1. (Color online) Transmission coefficient of a single circle hole in a Ag film $(t=340 \mathrm{~nm}$ ). (a) Transmission of single hole with $d=250 \mathrm{~nm}$ (solid line) and $d=300 \mathrm{~nm}$ (dash dot line) from experiment (Ref. 2), with $d$ $=250 \mathrm{~nm}$ (dash square line) and $d=270 \mathrm{~nm}$ (dash circle line) from FDTD calculation, with $d=270 \mathrm{~nm}$ from the ideal waveguide theory (thick solid line), and from Bethe approximation (dot line). The enhancement factor (dash line) is presented by dividing transmission from calculation by that of the waveguide theory. The arrows indicate the positions for different cylindrical surface plasmon excitation modes. Notice that similar oscillations appear in the experiment data with always a little shift. (b) Transmission of holes with $d=200 \mathrm{~nm}$ from experiment (solid line) (Ref. 2), from FDTD calculation (dash circle line), from the ideal metal waveguide theory (thick line), and from Bethe approximation (dot line). The enhancement factor, 10000 (dash line), is presented by dividing transmission from FDTD calculation by that for the waveguide theory.

seen, the agreement is astonishing and remarkably good without any fitting of parameters. The only input in the calculation is the frequency dependent permittivity as given by the experimental data by Johnson and Christy.

In order to understand the transmission spectrums that we have both from the experiments and the calculations, we should first take a look at the transmittivity of a cylindrical waveguide in an ideal metal, which shows a cut-off wavelength corresponding to $2 \pi d / 3.68$. $^{10}$ Consequently for $\lambda$ larger than this cut-off wavelength, there will be a fast exponential decay of the transmission as plotted in Figs. 1(a) and 1(b). This result excludes the existence of surface plasmons in the cylinder, because the waveguide theory for this cut-off mode requires the field in the cylindrical surface to be zero. However, the experiment and calculation confirm that there is much transmission intensity for $\lambda$ larger than the cut-off wavelength.

The explanation to this large transmission by a single hole has to be searched in the earlier papers on surface plasDownloaded 09 Mar 2010 to 161.111.180.191. Redistribution subje mons of cylinders (see Refs. 5 and 6 by Economou and coworkers) and is very easy to understand. The surface plasmons locate along the external circumference of the cylinder with possible wavelength $\lambda_{n}^{\theta}=2 \pi r / n$, i.e., circumference length divided by the index branch $n$. The wave propagates along the cylinder axis $z$ with a wave vector $k_{z}$. The possibility of exciting long wavelength modes is given by the cylindricality $\alpha=2 \pi r / \lambda_{p}$, where $\lambda_{p}$ is the bulk plasmon wavelength (in the case of $\mathrm{Ag}$, it is $325 \mathrm{~nm}$ according to experiment data in Ref. 7). In our case we do not have a cylinder of infinite length as has been discussed in Refs. 5 and 6 , but we have a cylindrical metallic cavity of certain thickness $t$. However, by looking at the boundary conditions the same kind of modes should exist. From our full solution of Maxwell equations, the modes show up in the transmission. In Fig. 1(a), the peaks in the transmission can be identified, which we believe correspond to the surface plasmon modes excited in the cavity's surface. The peak with longer wavelength corresponds to the smaller $n$. Notice in Fig. 1(a) the arrows indicating the oscillation peaks that are present in the transmission. The experiments manifested these oscillations as well. Also notice that a single hole presents enhancements of 10000 by comparing with waveguide theory, so that in the arrays the SPP excitation contribution to the enhancement is marginal. Also the calculations presented below show that for some, frequencies produce an enhancement but for others a depression.

Since a single hole gives such an enhancement over the cut-off wavelength, it will not be a surprise that a periodical array will also give a very large enhancement. The result for an array is produced by the interference of each hole's transmission and can be enhanced or depressed with respect to a single hole. Some ideas about this have been described in Ref. 2 In order to prove this we have performed FDTD calculations for a periodical array of holes to compare with the experiment data. ${ }^{2}$

Figure 2(a) shows a comparison of the results of experiments in Ref. 2 for periodical hole array with $P=600 \mathrm{~nm}$, $d=250 \mathrm{~nm}$, and $t=340 \mathrm{~nm}$. And again the agreement is remarkable in the peak positions, intensities, and enhancement with respect to a single hole intensity. We are actually performing calculations for an infinite array of holes, while in the experiment the holes' arrays are finite. However, the experiments showed that arrays of $N \times N$ holes yield practically the same results for $N>9$, so the infinite array should give, as shown by the calculations, a good approach.

We would like to comment on the appearance of the peak positions from the periodical arrays. Once the cylindrical surface plasmons are excited at the surface, they will propagate with a decay length and if the film thickness $t$ is short enough, smaller than the decay length, there will be a comparatively large transmission per hole. These plasmons radiate waves at the surface and then interfere. The peak positions and their intensity are given by the value of the period $P$. We have checked this by performing calculations for different periods from 500 to $1200 \mathrm{~nm}$ (not presented here). The hole diameter intervenes in the peak intensities because when the maximum of the single hole intensity falls at the same position as the ideal interference peak then this interference peak shows a pronounced maximum. However, if these conditions do not match, the peak of the array is much smaller. As an illustration we present calculations in Fig. 2(b) for $d=200 \mathrm{~nm}$ and $P=600 \mathrm{~nm}$. It is clearly seen to AlP license or copyright; see http://apl.aip.org/apl/copyright.jsp 


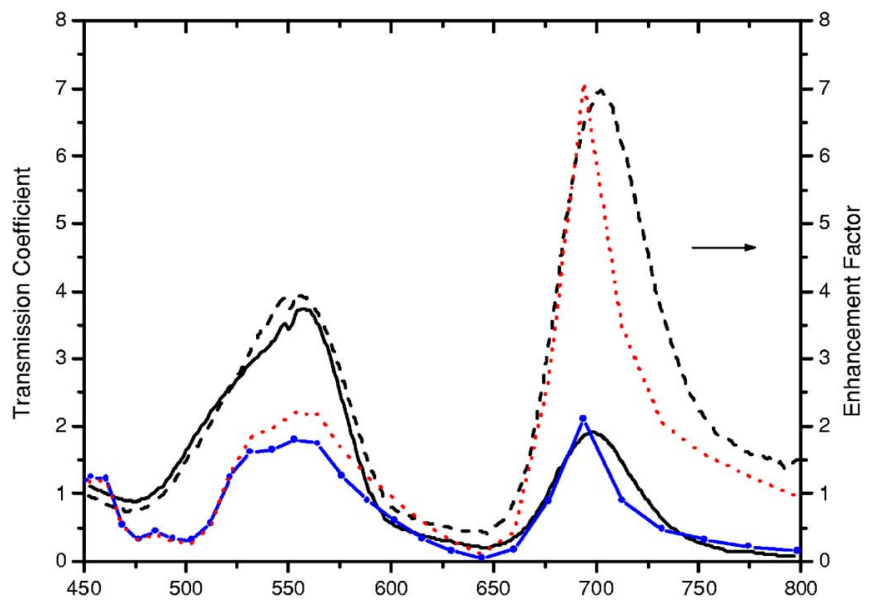

(a)

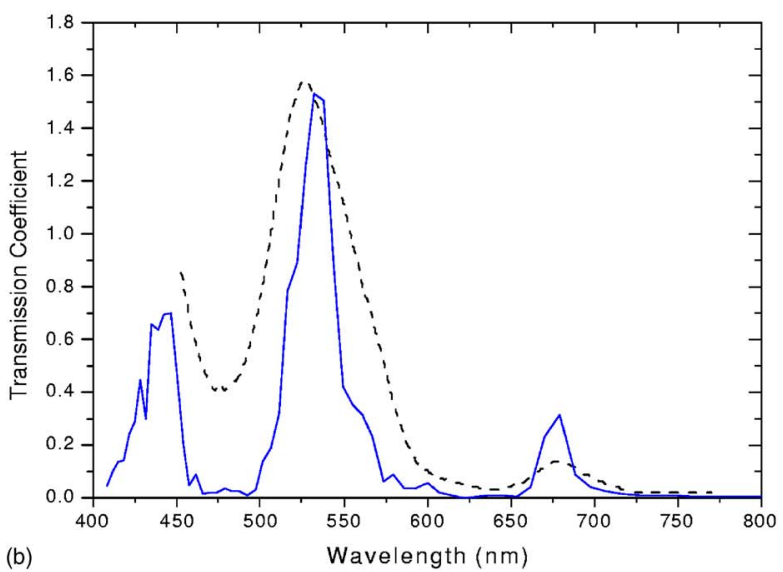

(b)

FIG. 2. (Color online) (a) Transmission coefficient of periodic array $(P$ $=600 \mathrm{~nm})$ of circle holes in a $\mathrm{Ag}$ film $(t=340 \mathrm{~nm})$ with $d=250 \mathrm{~nm}$ from the experiments (solid line) and the corresponding enhancement factor (dash line) [Figs. 2(a) and 2(b) in Ref. 2], together with the coefficient from our FDTD calculations (dash circle line) and the corresponding enhancement factor (dot line). (b) Transmission coefficient of periodic array $(P$ $=600 \mathrm{~nm})$ of circle holes in a Ag film $(t=340 \mathrm{~nm})$ with $d=200 \mathrm{~nm}$ from the experiments (dash line) [Fig. 2(c) in Ref. 2] and from our FDTD calculations (solid line)

that the enhanced peak at around $\lambda \approx 690 \mathrm{~nm}$ is strongly reduced because the single hole at this wavelength has little intensity (see Fig. 1). This is also in excellent agreement with the data of Ref. 2 Moreover, the thickness $t$ may also count, because the material has absorption and the plasmons have certain decay length. Actually, the propagation length, the plasmon speed, the retardations, etc. ${ }^{11}$ all play a role that needs to be investigated by changing the hole thickness and diameter systematically. We predict that by making the value of $t$ twice as large, the intensity will be highly reduced. Therefore the experiments ${ }^{1-3}$ have no extraordinary transmission and can be understood by the transmission of a single hole.

Another comment that should be addressed is that the model used by Economou and co-workers ${ }^{5,6}$ is for Drude dispersion. $\mathrm{Ag}$, gold, and copper, in the range of frequencies described here, can be approximately described by the plasmonic model. However, for the range of experimental frequencies $400<\lambda<900 \mathrm{~nm}$ the explanation of the cylindrical plasmons cannot be easily extended to other materials such as tungsten, because at these frequencies, it is a dielectric. ${ }^{8}$ But even if it is a dielectric, the peak positions of the hole array transmission are just given by the period; however, the peak intensities are smaller than for Ag. Preliminary calculations showed agreement with experiments of Ref. 2

We propose to do experiments for single holes, as well as arrays, changing systematically the values of $P, d$, and $t$ for these different materials. These will afford the values of decay length of propagation, the degree of confinement, as well as the speed of the waves. ${ }^{11}$

The authors thank the European EU-FP6 Project Molecular Imaging, LSHG-CT-2003-503259 for support.

${ }^{1}$ T. W. Ebbesen, H. J. Lezec, H. F. Ghaemi, T. Thio, and P. A. Wolff, Nature (London) 391, 667 (1998).

${ }^{2}$ H. J. Lezec and T. Thio, Opt. Express 12, 3629 (2004).

${ }^{3}$ H. Raether, Surface Plasmons on Smooth and Rough Surfaces and on Gratings, Springer Tracts in Modern Physics Vol. 111 (Spinger, Berlin, 1988), p. 92; N. García, Opt. Commun. 48, 1045 (1983); N. García, G. Diaz, J. H. Saenz, and C. Ocal, Surf. Sci. 143, 342 (1984).

${ }^{4}$ H. A. Bethe, Phys. Rev. 66, 163 (1944).

${ }^{5}$ C. A. Pfeiffer, E. N. Economou, and K. L. Ngai, Phys. Rev. B 10, 3038 (1974).

${ }^{6}$ S. S. Martinos and E. N. Economou, Phys. Rev. B 24, 6908 (1981).

${ }^{7}$ P. B. Johnson and R. W. Christy, Phys. Rev. B 6, 4370 (1972).

${ }^{8}$ J. H. Weaver, C. Krafka, D. W. Lynch, and E. E. Koch, FachInformations-Zentrum, Energie Physick Mathematik GmbH Physik Daten/ Physics Data No. 18-1, 1981 (unpublished).

${ }^{9}$ A. Taflove, Advances in Computational Electrodynamics, The FiniteDifference Time-Domain Method (Artech, House, Boston, 1998), p. 263.

${ }^{10}$ John David Jackson, Classical Electrodynamics, 3rd ed. (Wiley, New York, 1999), p. 362.

${ }^{11}$ M. Bai, C. Guerrero, S. Ioanid, E. Paz, M. Sanz, and N. García, Phys. Rev. B 69, 115416 (2004). 